\title{
Basic neurourology
}

\author{
Kwang Taek Kim', Hyun-Kyung Chang', Chang-Hee Kim', Kyung Jin Chung' ${ }^{1}$, Suzana Brown², Su Jin Kim³ , Khae-Hawn Kim,** \\ 'Department of Urology, Gil Medical Center, Gachon University College of Medicine, Incheon, Korea \\ 2Department of Technology and Society, The State University of New York (SUNY) in Korea, Incheon, Korea \\ ${ }^{3}$ Department of Urology, Yonsei University Wonju College of Medicine, Wonju, Korea
}

The integrated coordination of the components of the lower urinary tract is mediated by the complex neuromodulatory system of the brain, spinal cord, and peripheral ganglia. Therefore, the central nervous system plays a crucial role in the storage and output of urine. The purpose of this review article is to present the key aspects of the structure of the peripheral nervous system and central nervous system related to the lower urinary tract, as well as the mechanisms of action and the control system of innervation regulating the storage and output of urine. Furthermore, this article discusses the clinical significance and directions of neurourological research, concluding with the suggestions for with the neurourological research prospects.

Keywords: Neuromodulation, Lower urinary tract, Neurourology

\section{INTRODUCTION}

The axon bundles passing through the spinal cord from the cerebral cortex descend through the pyramidal tract and are divided into the lateral and anterior (or ventral) tracts inside the spinal cord, forming the corticospinal tract (Austin et al., 2014). Upper motor neurons (UMNs) are neurons with axons running to the spinal cord through the brain stem; their cell body is located in the motor cortex of the cerebral cortex, and they commonly do not come into direct contact with muscles (Han et al., 2017). Lower motor neurons (LMUs) are connected to UMNs in the spinal cord. Although they are usually connected by interneurons, in some cases UMNs and LMNs synapse in the anterior or ventral horn of the spinal cord (Fowler et al., 2008). The axons of LMUs pass through the efferent anterior or ventral roots, where motor nerves and autonomic nerves run when they exit the spinal cord. At that point, LMUs extend to the neuromuscular plate or neuromuscular junction, where they are responsible for innervation at muscles that are under voluntary control (Lee et al., 2018). Onuf's nucleus is a defined group of neurons that maintain continence of micturition and defecation and induce muscle contraction during orgasm. These neurons are located in the ventral part of the anterior horn in the sacral spinal cord. Notably, this part that, includes motor neurons, forms the pudendal origin of the perineal nerves (de Groat et al., 2015).

\section{INNERVATION OF THE LOWER URINARY TRACT}

The spine is composed of 26-30 vertebrae, which are divided into cervical, thoracic, lumbar, and sacral vertebrae. The thoracolumbar region of the spine comprises T10-L2, while the sacral region comprises S2-4. Each of these regions is composed of sympathetic neurons, parasympathetic neurons, and Onuf's nucleus, a special group of somatic nerves of interest for neurourology. The motor neurons in this nucleus predominantly innervate the external rhabdosphincter, which is under voluntary control. Motor neurons with a distinct mechanism of synaptic signal transmission control the ischiocavernosus and bulbocavernosus muscles, which are involved in penile erection and ejaculation. Even though these neurons participate in the process of signal transmission through a similar basic mechanism as other neurons_-via neurotransmitters
*Corresponding author: Khae-Hawn Kim (iD https://orcid.org/0000-0002-7045-8004 Department of Urology, Gil Medical Center, Gachon University School of Medicine, 21 Namdong-daero 774beon-gil, Namdong-gu, Incheon 21565, Korea

E-mail: kimcho99@gilhospital.com

Received: November 25, 2019 / Accepted: December 15, 2019
This is an Open Access article distributed under the terms of the Creative Commons Attribution Non-Commercial License (http://creativecommons.org/licenses/by-nc/4.0/) which permits unrestricted non-commercial use, distribution, and reproduction in any medium, provided the original work is properly cited. 
that are secreted by the presynaptic neuron and bind with postsynaptic receptors-their axons contain less-dense core vesicles and secretory granules than those found in autonomic nerves (Tai et al., 2009). The motor neurons of these nuclei are rich in serotonin (5-HT) and norepinephrine (NE) receptors and are activated by L-glutamate, a neurotransmitter that is the anionic form of glutamic acid. If activated by 5 -HT and NE, the guarding reflex, which suppresses urination, occurs and prevents urination when a sudden rise of abdominal pressure occurs. Concerning incontinence, a three-layer muscle is involved in urine flow and continence that consists of an inner band of longitudinal smooth muscle, a middle band of circular smooth muscle, and a striated muscle known as the outer band of the rhabdosphincter. This muscle is dominated by sympathetic neurons, parasympathetic neurons, and the somatic branch of the peripheral nervous system.

First, sympathetic neurons are distributed in the longitudinal and circular smooth muscle layers, running along the hypogastric nerve and synapsing with sympathetic preganglionic neurons located in the upper lumbar spinal cord (de Groat et al., 2015). Second, parasympathetic neurons arise from parasympathetic preganglionic neurons located in the sacral spinal cord and are also distributed in the longitudinal and circular smooth muscle layers. Finally, somatic nerves originate from motor neurons located in the ventral or anterior horn of the sacral spinal cord, which is known as Onuf's nucleus. The pudendal nerve also extends from Onuf's nucleus and directly controls the rhabdosphincter muscle, a sphincter consisting of striated muscle fibers. The sympathetic storage reflex, which is also called the pelvic-to-hypogastric reflex, starts when NE is secreted in response to bladder stretching (Vizzard, 2006). The previously mentioned somatic storage reflex, also known as the pelvic-to-pudendal or guarding reflex, can occur in situations such as laughing, sneezing, and coughing that induce a rise in vesical pressure due to increased abdominal pressure or detrusor pressure. L-glutamate, which is the primary excitatory transmitter in the reflex arc, triggers action potentials by stimulating $N$-methyl-D-aspartate, $a$-amino-3-hydroxy-5-methyl4-isoxazolepropionic acid, and transmembrane ionotropic receptors, and these action potentials stimulate the secretion of acetylcholine, thereby causing contraction of rhabdosphincter muscle fibers (Lee et al., 2018). The urinary incontinence occurs because of this stress if this guarding reflex does not function properly.

The neurological coordination of the lower urinary tract can be analyzed from the perspective of motor neurons or sensory neurons. Initially, sensory nerves with receptors in the bladder and urethra transmits stimuli to the cerebral cortex through the peri- aqueductal gray of the midbrain. Upon the recognition of stimuli, the cerebrum executes decision-making in response. Motor neurons are divided into UMNs and LMUs, and UMNs coordinate storage and urination in the brainstem for synergic voiding (Merrill et al., 2013). In contrast, LMUs, which originate in the spinal cord, cause muscles to contract. These neurons are present in the sacrum, and Onuf's nucleus is responsible for the contraction of the external urethral sphincter and maintains continence in states of rising vesical pressure through voluntary contraction of the sphincter. Parasympathetic neurons originating from S2-4 are responsible for the contraction of bladder muscles, while sympathetic neurons are responsible for contraction of the urethral smooth muscle, including the bladder neck, during the guarding reflex.

\section{CLINICAL SIGNIFICANCE}

Generally, in normal conditions, UMNs maintain a neuro-physiological state of LMNs suppression. Therefore, lower spinal cord injuries or peripheral nerve injuries can result in LMN dysfunction, leading to urinary retention, stress urinary incontinence, or poor compliance. On the other hand, upper spinal cord injuries or cerebral injuries that induce UMN dysfunction can lead to detrusor-sphincter dyssynergia, autonomic dysreflexia, and detrusor overactivity. In cerebral injuries that induce sensory neuron dysfunction, problems such as urinary retention can occur because of an inability to start urination caused by an inability to relax the rhabdosphincter muscle, which in turn stems from a lack of recognition of bladder filling and urinary flow in the urethra. Alternatively, disorders of decision-making, enuresis, nocturia, or urgency incontinence can occur due to a failure to correctly determine the appropriate time and place for urination. Fundamentally, the innervation of the lower urinary tract can be considered from multiple perspectives, and the first treatment principle in neurourology is the preservation of renal function and prevention of autonomic dysreflexia, followed by accomplishing improvements in storage and voiding symptoms.

\section{DIRECTIONS OF NEUROUROLOGICAL RESEARCH}

In the aging process or because of disease accompanied by neurological disorders, the cerebral control of suppression is weakened due to unnecessary activation of the micturition center. Nocturnal enuresis and accompanying lower urinary tract symptoms are sometimes thought to be caused by spinal reflexes that are not 
suppressed and controlled in the cerebrum. Therefore, if external local effects on inappropriate activation of the micturition center in the brain can be modified, these patterns of inappropriate activation could be controlled, and may even be possible to treat successfully (Salazar et al., 2017). If confirmed, this could be a promising possibility for our understanding of diseases accompanied by dysuria, such as aging and degenerative brain diseases, and it may facilitate the development of novel drugs therapy. Currently, urodynamic studies are used for the definitive establishment of the phenotypic cause of disorders when diagnosing and treating patients with lower urinary tract dysfunction, including neurogenic dysfunction. Urodynamic studies facilitate determination of whether the phenotype is myogenic, urotheliogenic, or neurogenic, and if it concludes that is neurogenic, they can shed light on the location of the damage. Subsequently, any controllable pathophysiological factors related to the patient's condition could be identified and addressed as part of the treatment plan. However, this treatment strategy is only applicable to peripheral treatment targets. Therefore, an integrated research framework exploring the factors that hinder central inhibition in the micturition center, as discussed above, may lead to the identification of various methods to control factors that promote dysfunction. In other words, it is necessary to find mechanisms for exploring the neurogenic phenotype.

\section{NEUROUROLOGICAL RESEARCH PROSPECTS}

The current state of the art of research in this field includes functional imaging studies, research into specific brain areas (e.g., the micturition center), and studies of specific neurons and neuron groups that are related to urination. Furthermore, animal experiments have explored how the activated and inactivated states of specific areas are associated with normal urination and dysuria. However, the basic mechanisms underlying these relationships have not been identified, meaning that specific methods to control such changes have not been developed. Observing and testing the roles of specific neurotransmitters and neuropeptides in controlling urination in the brain by activating and suppressing synaptic connections will make vital contributions to research into basic mechanisms in the field of neurourology (Kim, 2017).

Additionally, the goals of neurourological research should include monitoring how specific neurons are associated with urination in real time; identifying how the activation and inactivation of neurons in specific areas influence actual urination in specific physiological conditions (e.g., aging, sleeping, or studying) or specific pathological states (e.g., spinal cord injuries, cerebral injuries, or degenerative brain diseases); and exploring whether specific

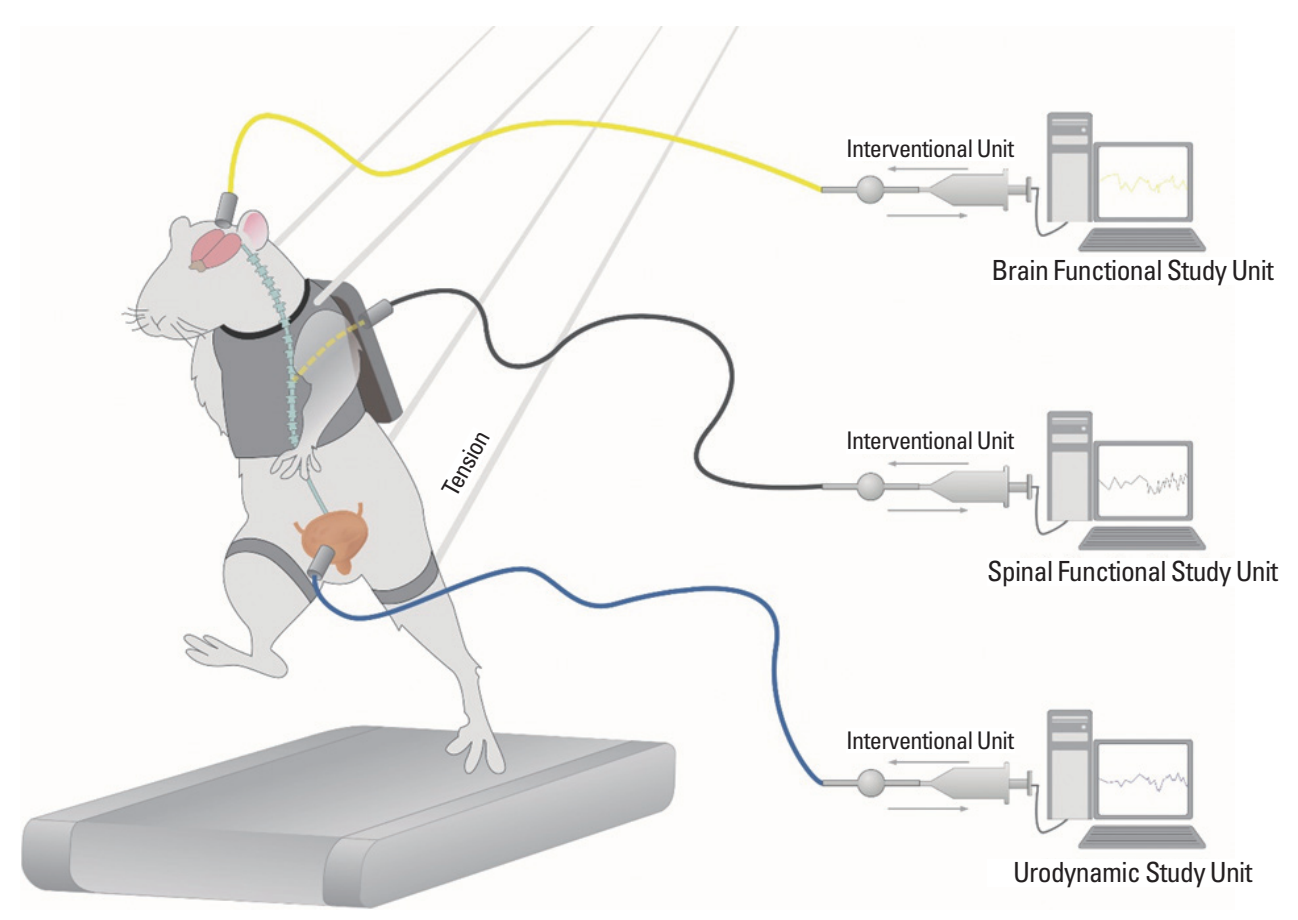

Fig. 1. The animal model used to obtain information for the diagnosis of voiding dysfunction and subsequent therapeutic interventions through functional behavioral studies. The interventional device includes a pressure transducer and a remote-controllable medicine injection or nerve stimulation system. 
treatments affect the activation level of neurons and how such influences are connected to changes in urination (Fig. 1).

\section{CONFLICT OF INTEREST}

No potential conflict of interest relevant to this article was reported.

\section{ACKNOWLEDGMENTS}

This study was supported by the Ministry of Education of the Republic of Korea and the National Research Foundation of Korea (NRF-2017R1A2B2005412).

\section{REFERENCES}

Austin PF, Bauer SB, Bower W, Chase J, Franco I, Hoebeke P, Rittig S, Vande Walle J, von Gontard A, Wright A, Yang SS, Nevéus T. The standardization of terminology of lower urinary tract function in children and adolescents: update report from the Standardization Committee of the International Children's Continence Society. J Urol 2014;191:18631865.e13.

de Groat WC, Yoshimura N. Anatomy and physiology of the lower urinary tract. Handb Clin Neurol 2015;130:61-108.
Fowler CJ, Griffiths D, de Groat WC. The neural control of micturition. Nat Rev Neurosci 2008;9:453-466.

Han JH, Kim SE, Ko IG, Kim J, Kim KH. Afferent pathway-mediated effect of $\alpha 1$ adrenergic antagonist, tamsulosin, on the neurogenic bladder after spinal cord injury. Int Neurourol J 2017;21:178-188.

Kim KH. Expected Next-Generation Drugs. Int Neurourol J 2017;21:81-82. Lee JM, Kim TW, Park SS, Han JH, Shin MS, Lim BV, Kim SH, Baek SS, Cho YS, Kim KH. Treadmill exercise improves motor function by suppressing purkinje cell loss in parkinson disease rats. Int Neurourol J 2018;22(Suppl 3):S147-155.

Merrill L, Girard B, Arms L, Guertin P, Vizzard MA. Neuropeptide/receptor expression and plasticity in micturition pathways. Curr Pharm Des 2013;19:4411-4422.

Salazar BH, Hoffman KA, Zhang C, Kavanagh A, Zhang Y, Boone TB, Munoz A. Electrical activity of the bladder is attenuated by intravesical inhibition of $\mathrm{P} 2 \mathrm{X} 2 / 3$ receptors during micturition in female rats. Int Neurourol J 2017;21:259-269.

Tai C, Wang J, Jin T, Wang P, Kim SG, Roppolo JR, de Groat WC. Brain switch for reflex micturition control detected by FMRI in rats. J Neurophysiol 2009;102:2719-2730.

Vizzard MA. Neurochemical plasticity and the role of neurotrophic factors in bladder reflex pathways after spinal cord injury. Prog Brain Res 2006;152:97-115. 